\title{
The Management of Age Related Cataract in Sub-Saharan Africa
}

\author{
Lawan Abdu \\ Department of Ophthalmology, Faculty of Medicine, Bayero University Kano \\ Nigeria
}

\section{Introduction}

Cataract simply means opacity of the crystalline lens. The human lens is essentially an ectodermal structure and develops from the lens placode about the fifth week of gestation. The lens has a capsule formed early in the gestation period. This makes lens protein isolated from the human immune system ab-initio. The lens grows through out life from about 90 grams at birth to 250 grams in weight at the age of sixty years. The equatorial diameter is 9 to $10 \mathrm{~mm}$ in adults. It is an avascular structure and obtains nutrition from surrounding vitreous and aqueous humor. Metabolic processes include the hexose monophosphate, the pyrubic and the aldose reductase pathway which produce sorbitol. The active substance produced by the numerous pathways is glucose 6 phosphates essential for generating energy for the lens metabolism. Fluid movement in the lens is mediated by $\mathrm{Na}+\mathrm{K}$ ATpase. The lens structure consists of a nucleus with two " $Y$ " shaped sutures, erect anteriorly and inverted posteriorly. This is surrounded by the cortex. The epithelium lies under the capsule except posteriorly. The lens function includes accommodation and contributes one third of the refractive power of the human eye being second to the cornea in this role. Cataract formation is an age related change that occurs from about the age of forty years. Various mechanisms have been advocated as the cause of this opacification notably oxidative damage by free radicals generated during normal metabolic activity and 300 to $400 \mathrm{~nm}$ radiation present in the natural human environment. There are lens enzymes that function to protect against free radical damage such glutathione peroxidase, catalase and superoxide dismutase. The level of these enzymes is reduced in the lens of patients who develop age related cataracts. There are various morphological types of age related cataracts. The location of the opacity defines the morphological appearance and this may be nuclear, sutural, cortical sub capsular (anterior or posterior), and or, capsular. Cataract can be described as immature, mature or hyper mature based on the density of the opacification. The location of the cataract has a role in determining the extent, type and severity of the symptoms. A cataract causes painless progressive loss of vision. Ordinary, it is expected that the symptoms parallel the degree of loss of lens transparency. This is not entirely true as even a smaller opacity on the visual axis particularly when close to the nodal point of the eye as seen in posterior sub capsular cataract can cause significant visual disability irrespective of extent of opacification. The nature of day to day life of the individual and 
occupational demands can contribute to the patient's awareness of visual limitation even at an early stage. Age related cataract is one of the leading causes of avoidable or curable blindness worldwide and affect half of the estimated forty five million world's population who are blind. It also causes visual impairment in many others. There is no gender predilection. In developing countries of Africa south of the Sahara, the problem is more severe and the impact on the life of the people is tremendous. Many factors contribute to the large burden of cataract blindness in this part of the World. There is a huge underserved population. Ophthalmologists are very few in numbers with their ratio to the general population are about one to a million. The eye care workers largely concentrate in urban areas whereas seventy percent of the population lives in the rural areas. Most of the trained eye care professionals are not in public service and only the rich can afford to pay for private eye care. There is high level of poverty in this part of the world. Most of the populations are small scale farmers who toil during the rainy season to harvest food that can barely feed the large families. Blindness thus contributes to loss of productivity. In the event of a family member becoming blind the younger children are made to leave school and serve as guide to the disabled adult. This further perpetuates illiteracy, poverty and deprivation. There is cost attached to treatment in the cities for the few who eventually make it there. This is about three times the cost of a bag of corn that families eat over several months. Most public eye hospitals have a waiting list and there is need for improvement in the professionalism of some providers and quality of the services provided. Outreach services are infrequent, poorly sustained and often defend on Non Governmental Organizations (NGO's) based in other countries. Where eye camp services are provided initial evaluation and monitoring at follow up are inadequate. Quacks who masquerade as traditional eye care practitioners abound. Their role is to compound and limit the success of the few hard working trained and registered eye care providers. The trade mark of the traditional eye healers (TEH) is sale to unsuspecting, unassuming, desperate and gullible public of traditional eye medicine (TEM), the nature, content, dosage and ill effects of which is not known even to the vendors of these concoctions. TEM can cause corneal damage and thus blindness and severe visual impairment. Harmful traditional eye practices such as couching results in severe ocular inflammation, hypheama, secondary glaucoma, endophthalmitis and retinal detachment. The activities of TEH are unregulated and they often enjoy patronage of government health departments particularly at district level. Their "successes" in plying their trade may not be unrelated to financial understanding with district health officials in some countries. In this chapter, the practice of cataract surgery in this part of the world is described to further the understanding of surgeons from other parts of the world who may wish to compare to that widely used in sub-Saharan Africa as described in this chapter.

\section{Pre operative management of patient with age related cataract}

The commonest symptom of age related cataract is painless progressive visual loss. Vision is often described by patients as "smoky" or "hazy" and likened to the environment as it appears during the harmattan season when cold dusty winds blow southwards across the Sahara Desert. Patient evaluation is the most essential step in offering quality eye care. A careful history should include duration and possible interventions tried by the patient. 
Presence of other symptoms such as pain may indicate that cataract is associated with other co existing ocular morbidity. Intermittent blurring of vision, seeing rainbow like colors may indicate associated glaucoma co existing or as a differential diagnosis. A history of application of harmful traditional eye medications or traditional eye surgery is important as this may influence the treatment option to be offered. Likewise there is need to ask of history of any medical illnesses particularly diabetes mellitus and hypertension. It is important to know duration of these diseases and treatment offered including compliance with medication as retinal complications of diabetes mellitus are partly time related. Assessment of the patient includes basic eye examination notably, unaided and pin hole visual acuity. A fully matured cataract usually cause blindness or visual impairment without improvement in pin hole vision. It is not unusual to see patients with bilateral cataracts. The cornea is usually clear in patients with cataract despite the prevalence trachoma presence of opacity is a hall mark of possible attempted couching. The anterior chamber is usually deep and quite. Presence of activity may denote reaction to application of TEM or other cause of inflammation in the anterior segment which may be lens related. Brisk pupil reactions indicate possible absence of gross retinal and optic nerve disease. Poor or absent pupillary reaction to both direct and consensual stimuli should alert the clinician to other causes of vision loss apart from age related cataract. All patients with cataract must have intra ocular pressure measured preferably with an applanation tonometer which is the gold standard. Raised intra ocular pressure (IOP) may indicate co existing glaucoma and thus patient need to have the optic disc evaluated with indirect ophthalmoscope to obtain a clear fundal view after pupil dilation, gonioscopy and also assessment of the contra lateral eye for possible evidence of glaucoma. Various ways of grossly assessing the retina includes the four quadrants light projection test, two-point light discrimination test at, 5, 7 and 12 centimeters from the eye for patients with light perception, hand motion and counting finger vision, respectively. Ophthalmic B mode ultrasound scan can be performed when there is suspicion of retinal detachment. Laser interferometry through selected areas of the cataract to assess retinal function can be applied where such facilities are available. Routine blood pressure measurement, full blood count and fasting blood sugar are advised for all patients in this part of the world. Biometry is performed to determine the power of intra ocular lens required to achieve target post operative visual acuity. To prepare patient for surgery there is need to administer and obtain an informed consent. Preoperative trimming of eye lashes and administration of $500 \mathrm{mg}$ of systemic acetazolamide is desirable by ophthalmologist in this part of the world. The surgery of choice in most centers is extra capsular cataract extraction with posterior chamber intra ocular lens implantation (ECCE + PCIOL).

\section{Indication for cataract surgery}

Poor vision is the commonest indication for removal of cataract. Cataract is the most cost effective surgical intervention. Successful cataract surgery almost gives instantaneous results and restores the patient back to his normal life style. Other reasons for performing cataract surgery are when the presence of the cataract is impeding the health of the eye such as in anteriorly dislocated cataract, intumescent cataract and phacolytic glaucoma. Cataract removal may be required to visualize the posterior segment. Rarely a white cataract is removed in otherwise blind eye to restore black pupil. 


\section{Anesthesia for cataract surgery}

Both general and regional anesthetic block can be used in patients with age related cataracts. However, most cataract operations are performed under local anesthesia. The aim is to provide pain relief for the patients comfort and compliance during the procedure. Akinesia help ophthalmologist to perform the surgery. There are various ways of ensuring lid akinesia. The $\mathrm{O}^{\prime}$ Brien technique blocks the facial nerve around the neck of the mandible. Van Lint's block is of the nerve under the periusteum at the lateral orbital rim and Atkinson's block is applied mid way between these two. The commonly used anesthetic is Lignocaine (xylocaine) mixed with adrenaline (1:100000 dilutions). The anesthetic agent can be injected into the muscle cone behind the globe. The technique is termed the retro-bulbar retro-ocular block though carry a higher risk of globe penetration. This risk is reduced with other para-bulbar techniques relying on injecting relevant areas such as para-bulbar or sub-Tenon's block. Topical anesthesia is rarely practiced in subSaharan Africa as most patients tend to have a low pain threshold. The advantage of using lignocaine with adrenaline is that it causes local vasoconstriction ensuring greater effect of the anesthesia and limiting systemic absorption. It can be used alone or with hyaluronidase (healon) to further aid in local distribution and absorption. Xylocaine effect lasts up to two hours and may be mixed with mupivacaine (marcaine heavy) the effect of the later starting within two hours and lasting longer. 2 to 3.5 milliliters can be injected into the retro bulbar space and 5 milliliters is used for facial nerve block. There is need for caution as large quantities can raise the intra orbital pressure (and thus the intra ocular pressure) which makes it more difficult to handle the globe during surgery. The choice of type of regional block partly defends on the circumstances, the patient and the choice of the ophthalmologist. Retro-bulbar injection is not risk free and there are complications in addition to accidental perforation of the globe. Orbital hemorrhage can cause instantaneous proptosis warranting deferral of surgery and may require urgent orbital decompression. Lignocaine used in this procedure can enter the sub arachnoids space and track to the base of the brain interfering with the function of the cardiovascular control mechanism giving raise to fall in systemic blood pressure and hypovolaemic shock. There is need for caution in this procedure and vigilance to ensure prompt intervention when any complications arise. Patients must fast overnight and should not eat on the day of the surgery so that conversion from local to general anesthesia can safely be effected should there be a need to do so. Patients on treatment for other systemic diseases such as diabetic and systemic hypertension must have there medications continued or adjusted in collaboration with the relevant physician.

\subsection{Intra capsular cataract extraction with correction of aphakia}

In the early part of the nineteenth century Duvial introduced intra capsular cataract extraction (ICCE) using the ab interno limbal incision approach. The aim is to extract the opaque lens with its capsule intact and remove it from the eye. This relatively simple procedure has been practiced in most countries of sub \Saharan Africa until conversion to extra capsular cataract extraction with posterior chamber intra ocular lens became more wide spread in the mid 1990. This procedure is performed under complete asepsis. After regional anesthetic block, the operation site is cleaned and draped. A speculum is applied to 
open the lids. A superior rectus bridle suture is applied to stabilize the eye ball. A number 15 or 11 surgical blade is used to make a 180 degrees superior limbal incision. Peripheral iridectomy is done routinely to avert some of the complications associated with this procedure. The lens is delivered using vectis loop applied to the proximal lip of the limbal wound and a muscle hook gently applied to the sclera at $6 \mathrm{o}^{\prime}$ clock position to maneuver the lens through the open wound site. The limbal wound is the closed with interrupted $8 / 0$ silk sutures. The anterior chamber reformed with normal saline or Ringer's solution. Sub conjunctival steroid and antibiotic injection is then administered. Intra operative Atropine $1 \%$ eye drop is applied and the eye padded.

\subsection{Intra operative complications of intra capsular cataract extraction}

Vitreous loss: This complication is relatively common with ICCE and warrants vitrectomy. An anterior vitrector may be used, assisted by administration of intra-ocular triamcenolone. Alternatively, if not available the anterior vitreous surface is lifted with a cell sponge and cut with de Wecker scissors possibly aided Vannas scissors. Poorly managed vitreous loss can lead to retinal detachment, and also an up drawn pupil thus reducing the efficacy of any aphakic correction given to the patient later. Often an inferior sphinterotomy is performed to keep the pupil central. The anterior chamber can be reformed with sterile air to keep the vitreous back. The air is eventually absorbed in a day or two.

Intra operative hypheama: Bleeding into the anterior chamber during surgery can easily track down into the vitreous as there is no posterior capsule to serve as a barrier. Blood in the vitreous compromise the visual outcome. Significant amounts of blood in the vitreous can take up to a year to be absorbed and can be a source of fibrous tissue reaction. It can warrant pars plana vitrectomy if a retinal break cannot be excluded on fundoscopy. Any hyphema is immediately and promptly aspirated and the cause of the hemorrhage identified and treated.

Lens dislocation into the vitreous: Occasionally the lens can dislocate into the vitreous as the zonules are weak with age. Caution is necessary as the lens is carefully extracted from the eye.

Other complications: This includes corneal decompensation, descemet membrane stripping and expulsive choroidal hemorrhage.

\subsection{Post operative complications of ICCE}

Wound dehiscence: This is characterized by shallow anterior chamber and a positive Seidel's test. Padding and bandaging with a contact lens can aid sealing of small opening. Secondary wound closure may be necessary when there is significant wound break down.

Secondary Glaucoma: Secondary glaucoma can occur after intra capsular cataract extraction. Malignant glaucoma arises when aqueous humor track behind the vitreous, pushes the vitreous forward resulting in shallow anterior chamber with raised intra ocular pressure. The condition is treated with oral or intra venous acetazolamide. Patient may require vitrectomy to alter the misdirected aqueous humor. Phacolytic glaucoma is said to arise with the natural immune system react to leaked lens protein. This can occur when there is rapture of the lens capsule or when there is previous exposure of lens matter in the contra lateral eye. Treatment involves the use of topical steroids and anti 
glaucoma medication. Dislocation of the lens into the anterior chamber for any reason can lead to elevation in the intra ocular pressure and warrants immediate surgery to remove the lens.

Postoperative bacterial endophthalmitis: This is rare but a cause of great concern to ophthalmologists. Sterile endophthalmitis such as toxic anterior segment syndrome can be noticed within 24 hours after surgery and could be caused by the irritating substance in the surgeon's gloves. True bacterial endophthalmitis is seen within 24 to 72 hours after surgery. It is characterized by reduction in vision and pain. The lid appears swollen and the eye is injected. There is reduction in the post operative visual acuity. Most often it is caused by Staphlococcus epidermidis and Staphylococcus aureus though pseudomonas and proteus species have also been implicated. If unattended the condition can rapidly deteriorate leading to panophthalmitis warranting evisceration of the eye. Management includes prompt diagnosis and commencement of relevant topical and systemic antibiotics. Identification of the causative agent requires aqueous and vitreous aspirate for gram stain, culture and sensitivity tests. Intra vitreal antibiotic injections in standard dilutions are administered. Initial choice of antibiotic is modified based on final culture results. Some of the antibiotics used include gentamycin, amikacin, and vancomycin in standardized dilutions for intra vitreal and intra cameral administration. Systemic antibiotics are also administered such as intravenous ceftazidine and oral ciprofloxacin. Periocular antibiotic injections can also be administered. Adjuvant treatment includes cycloplegia and analgesia. Topical steroids may be introduced at a certain stage when infection is control. Steroids are given to reduce severity of the post inflammatory healing process as scars in the visual axis can also hinder the patient's vision.

Cystoid macula edema: This is otherwise called Irvine-Gass syndrome. Although the exact mechanism is unclear vascular incompetence and inflammation is a possible cause. CMO can be a complication of both ICCE and ECCE. Prophylactic administration of prostaglandin synthesases inhibitor such as indomethacin is said to reduce the risk.

Suture related complications: Epithelial in growth to the anterior chamber through the suture track can occur. Sutures can fragment or general fibrosis along its track. Meticulous wound handling is important in reducing such complications.

Retinal detachment: ICCE is associated with aphakic retinal detachment in up to $2 \%$ of cases. When there is vitreous loss the risk of retinal detachment is about $10 \%$. The presence of lattice degeneration, vitreous degeneration and pre existing high myopia are predisposing factors. Aphakic detachment requires identification of retinal breaks and vitrectomy or performing a buckling procedure, sealing of all retinal breaks and drainage of sub retinal fluid.

\subsection{Correction of aphakia after intra capsular cataract extraction}

Correction of aphakia is as important as removing the cataract. There are various ways of correcting aphakia; however the commonest method is by using spectacles. Spectacles for aphakic correction are available and affordable in most countries of the region. Disadvantage of spectacles include weigh, discomfort and the various spherical and chromatic aberrations resulting in poor compliance. High plus lenses causes scotoma which result in the 'Jack in the box' phenomenon. Pincushion distortion causes difficulty in the patient's general mobility particularly through doorways. With advancing age and 
confusion patients need to be educated on the use of such glasses to prevent falls and injuries. Glasses can be misplaced or loss, they get scratch marks and break. Aphakic spectacle lenses are not suitable for correction of uni-ocular aphakia. They cause a $25 \%$ image magnification beyond the $5 \%$ image differential which the brain can tolerate to ensure binocular single vision. These untoward effects warrant consideration of other methods of correcting aphakia. Contact lenses (CL) can be used for uni-ocular aphakia. CL is not affordable and unavailable in the rural settings. Their use requires high level of patient education and most of those who need it are semi literate at best. Soft extended wear CLs are rarely affordable and are effectively unavailable in rural settings. Soft extended ware CLs are very expensive and so their cleaning solutions. The living environment is dry and dusty further increasing the risk of corneal abrasion and secondary microbial infection that can result in cornel ulcer. For these reasons a contact lens will usually be a poor choice for correction for a farmer in the rural areas of sub Saharan Africa due to the numerous problems associated with their use. Secondary intraocular lens (IOL) procedures may be considered. Secondary anterior IOL implantation can be performed. Secondary posterior chamber (PC) IOL implantation tends to be more difficult to perform. Often surgeons avoid secondary IOL procedures particularly in patients with aphakia due to couching due to the high risk of retinal detachment. Photo refractive surgery such as epikeratopia (epikeratopia) and keratomileusis are rarely practiced. The skill and equipment required for LASIK and related modern procedures are simply unavailable. Gradually in the last decade or so extra capsular cataract extraction with PC IOL has largely replace ICCE.

\subsection{Extra capsular cataract extraction with posterior chamber intra ocular lens implantation}

The need to obtain quality post operative vision necessitates the conversion from ICCE to ECCE PCIOL. The IOL implant is within the eye and close to the nodal point hence simulating the role of the crystalline lens. Harold Ridley introduced the use of IOL in 1946 in United Kingdom after the Second World War. The IOL is widely accepted and is the gold standard of correcting aphakia in age related cataracts. The following steps are often observed in performing this procedure in sub-Sahara. The eye lashes are trimmed, $500 \mathrm{mg}$ of oral acetazolamide is given at least an hour before surgery to reduce the intraocular pressure and topical Atropine 1\% administered to dilate the pupil. Patients require biometry consisting of an ultrasound A scan and Keratometer to determine the power of the intra ocular lens required to give the desired post operative visual acuity. The target post operative visual acuity by most surgeons' is $6 / 6(20 / 20)$. The surgery is performed under complete aseptic condition. The steps involved in performing this procedure include cleaning with povidone iodine and draping. The lids are opened with a speculum wire. Superior rectus suture is placed to stabilize the eye. Fornix based conjunctiva flap raised.

\subsection{Techniques of approach into the anterior chamber}

Corneo scleral incision: From time immemorial surgeons are known to make limbal incision superiorly to gain access into the anterior chamber. This approach is quite simple and requires minimal tissue manipulation. Conventionally it is usually located 
between 10 o'clock and 2 o'clock position but may be modified in the presence of a filtering bleb.

Scleral incision: This is located $2-3 \mathrm{~mm}$ from the limbus. In the early days before minimal incision cataract surgery these incisions tended to be wide enough to allow the lens to be extracted during intra capsular cataract extraction. The size is relatively smaller in ECCE and even more so in small incision cataract surgery. The advantage is that it causes less post operative astigmatism than a corneoscleral incision. However it tends to bleed more than the former. With advances in cataract surgery scleral tunnel incision is the norm in most minimal access procedures in sub-Saharan Africa. In manual small incision cataract surgeries the opening is 3 to $4 \mathrm{~mm}$ and can be even less with phacoemulsification surgery and insertion of foldable intraocular lenses. Sclera tunnel incision give rise to less post operative astigmatism and do not require use of sutures to close the wound.

Corneal incision: This is performed at the superior corneal periphery using a razor blade fragment. The incision is beveled by design to offer a self sealing access though sutures are inserted at the end of the procedure. Corneal incisions can be performed in the presence of a filtering bleb. They cause no significant bleeding, but can lead to more post operative astigmatism particularly with less experienced surgeons. Wound closure can be by interrupted or continuous suture and the stump is buried in the corneal stroma to reduce irritation. The scar is small, parallel to the limbus and hardly noticeable as it is covered by the upper eye lid. The corneal incision has complications such as descemet stripping and can predispose to corneal decompensation. After gaining access into the anterior chamber (AC), viscoelastic material is injected to maintain the $\mathrm{AC}$ and protect the corneal endothelium from abrading by the surgeon's instruments.

Anterior capsulotomy: There are various techniques of performing capsulotomy, but the common practice is Can opener capsulotomy. Under visco elastic an angulated insulin needle is used to make small and continuous " $\mathrm{v}$ " shaped incisions on the peripheral lens capsule. The anterior capsule is then gently pickup with a capsule or Macpherson's forceps and removed through the wound site thus allowing for vectis nucleus delivery by counter pressure. Other modality includes continuous curvilinear capsulorheisis. The capsule is opened at one position and the torn in round and systematic manner till it is completely peeled off. There are various modifications such as envelope technique whereby the opening is shaped like an envelope. The aim is to provide access for removing the lens cortex and allowing for clean up of cortical matter.

Delivery of the nucleus: In ECCE after anterior capsulotomy, a vectis loop is applied to the proximal scleral side of the limbal wound and a squint muscle hook applied to the sclera at the 6 o'clock position. Gentle counter-pressure is applied to push the nucleus out through the wound edge. This technique is slightly modified in small incision cataract surgery. The symcoe aspiration-irrigation double canula is used to suck out as much cortex as possible using the vacuum in the handheld syringe; the nucleus is maneuvered into the anterior chamber before delivery through the wound site. Should the nucleus appear too large for the wound site, then it is fragmented with Macpherson's forceps into two in the anterior chamber before extrusion from the globe. Irrespective of the technique used, once the nucleus id delivered, the cortical matter is aspirated with irrigation-aspiration canula. This is performed with minimal disturbance to the iris as doing so can provoke more post operative inflammation. 
Insertion and positioning of the posterior chamber intra ocular lens: The anterior chamber is reformed with visco elastic material. A lens introducer is used to hold the IOL after the sterile lens pack is opened. The lens is introduced into the eye with the lower haptic pointing to the left of the surgeon. The lower haptic is inserted into the lower portion of the capsule (in the bag) or into the ciliary sulcus behind the iris. The upper haptic is then picked with Macpherson's forceps and gently pushed under the upper iris-pupil border associated with clockwise rotation thus slotting the lens into position. The IOL can then be rotated further by applying the dialer into the dialing hole at the periphery of the lens optic and rotating till the pupil is round and centered indicating that the IOL is properly positioned. The wound can then be closed with interrupted $9 / 0$ or $10 / 0$ silk sutures. The bite depth is two third the thickness of the cornea, the length of the scleral side of the suture is four time that of the corneal side. The sutures are radially oriented and evenly spaced to reduce post operative astigmatism. In small incision cataract surgery the scleral tunnel incision site is not sutured. Any remaining visco elastic material is aspirated from the anterior chamber. Subconjunctival injection consisting of gentamycin $20 \mathrm{mg}$ and dexamethasone $4 \mathrm{mg}$ is given to gently balloon the proximal end of the fornix based flap. Intra operative Tropicamide drop is administered and the eye is padded.

\section{Postoperative care of the pseudophakic eye}

Cataract surgery can be performed as a day case procedure. Mobile units such as are used in parts of India are not very common in sub-Saharan Africa. A significant proportion of patients come to the hospital from distant and remote places thus there is need for admission for a day or so. Postoperative care involves assessment of the visual acuity unaided and then with pin hole. Visual improvement usually is noted even from the first post operative day. Improvement with pin hole is unpredictable as the effect of cycloplegia is present a day after surgery. The wound site is inspected for any gaping in the wound. The cornea is inspected with the slip lamp. There may be edema and striate keratopathy. The anterior chamber may show flare and cells due to postoperative inflammation. Fundoscopy is performed- the fundus is usually normal though even then the view may appear blurred owing to corneal edema. Intra ocular pressure may be normal or elevated. Postoperative medication consists of topical steroids and antibiotics. Patients are followed up routinely and refraction performed after four weeks.

\subsection{Complications of extra capsular cataract extraction with posterior chamber intra ocular lens implantation}

A number of complications can occur which can pose special problems in the absence of nearby ophthalmic surgeon with one ophthalmologist to a million population in subSaharan Africa.

\subsection{Intra operative complications}

Posterior capsular tear: A tear or rent in the posterior capsule can occur. The posterior capsule serves as a scaffold upon which the PCIOL rests- a tear in the posterior capsule can alter the procedure. A small rent in the posterior capsule does not preclude insertion of the IOL which can be inserted in the capsular bag if there is enough support or in the ciliary 
sulcus. Large tears can necessitate more significant modification of the procedure, either non-insertion of the IOL or use of anterior chamber lens possibly as a secondary procedure. Large capsular rent can result in use of anterior chamber rather than posterior chamber intra ocular lens. High intra-orbital pressure which is transmitted to the intraocular pressure can make the ocular tissues more difficult to handle during surgery predisposing to posterior capsular tear. When surgery is performed under regional anesthesia, a restless and uncooperative patient is more likely to have a capsular rent than a more calm and cooperative one. Care of the posterior capsule is of paramount importance in providing a stable scaffold for the PCIOL.

Stripping of the descemet membrane: Occasionally the descemet is peeled usually from the lip of the limbal wound towards the center of the cornea. Visco elastic material can be injected from the center of the anterior chamber towards the stripped edge aiding its re positioning.

Intra operative hypheama: Bleeding into the anterior chamber can occur during surgery from tear in the iris root or anomalous blood vessel, often obscuring the operating view for the surgeon. A platelet plug promptly seal bleeding vessels, the blood is aspirated with Symcoe canula and procedure continued. It is rare for massive bleeding to occur which will warrant more drastic intervention.

Corneal decompensation: The cornea can suddenly become cloudy particularly with diseased endothelium such as guttata. It is therefore mandatory to examine the endothelium carefully before surgery. Care in avoiding contact between the endothelium and the operating instruments are mandatory in all patients.

Choroidal hemorrhage: Expulsive supra choroidal hemorrhage arises from rapture of the long or short posterior ciliary arteries and outcome unless action is immediately taken is expulsion of the contents of the eye. Many factors are associated with suprachoroidal bleeding including sudden fall in intraocular pressure, patient moving like coughing causing damage from instruments, vasalva maneuver, possible changes in blood pressure and use of retrobulbar anesthesia. Treatment includes mandatory immediate closure of the wound with firm sutures to tamponade the intraocular bleeding and some clinicians advocate immediate posterior sclerostomy. The latter can however compound the situation. After wound closure the patients is given one to two weeks before draining the accumulated blood. Some patients may require vitrectomy and air fluid exchange.

\subsection{Postoperative complications}

Wound leakage: Wound failure is one of the early complications in cataract surgery. It is easily detected by presence of excessive fluid (and may be noticed by the patients as excess tears) associated with the operated eye; the anterior chamber is shallow or may even be flat defending on the extent. Wound leak can be confirmed by applying fluorescein to the eye and examining with the cobalt blue light under slip lamp-the leakage site appears green as the fluorescein is diluted by leaked aqueous humor. Flourescein-impregnated strips can also be used. This use of flourescein is called Seidel's Test. Slight leakage can be sealed by bandage contact lens and if not available padding with firm bandage is used in sub-Saharan Africa. Huge leaks require secondary wound suturing. 
Iris prolapse: This can arise from poor wound closure and needs to be reposition and the wound closed properly. It can result in poor wound healing, postoperative astigmatism and increases the possibility of post operative endophthalmitis

Striate keratopathy: Is characterized by corneal edema and folds in the Descemet's membrane. Striate keratopathy is caused by damage to the corneal endothelium by inserted operating instruments. Care must be exercised during surgery to prevent endothelial cell loss. Viscoelastic material is used to protect the endothelium.

Postoperative inflammation (uveitis) and endophthalmitis: Sometimes the inflammatory response after surgery can be marked with fibrin formation. Minimal tissue manipulation results in less severe postoperative reaction. The severity is assessed with slit lamp examination. Medications consisting of anti-inflammatory, cycloplegics and intraocular pressure (IOP) lowering agents are prescribed. In some instances subconjunctival dexamethasone is administered. Patients require close monitoring till the process is fully controlled. Alternatively, endophthalmitis can occur, which demands intravitreal and possibly anterior chamber antibiotics to be administered as an emergency.

Raised intra ocular pressure: All patients must have the intra ocular pressure (IOP) measured postoperatively as the inflammation mentioned above can be associated with elevation in IOP. IOP-lowering drugs are administered when the need arise.

Residual cortex: Meticulous cortical clean-up is desirable though sometimes missed lens matter appears a day or two after surgery. This can cause more inflammation and the threshold for intervention by aspiration should be low.

Malposition of the intra ocular lens: Malposition lead to tilting of the lens from the most optimal position-this can lead to post operative astigmatism and lead to reduction in the patient's visual acuity. Malposition can result in distortion of the pupil which can appear decentered. Conservative management includes use of miotics. Occasionally, the lens has to be redialed into position.

Posterior capsular opacity (PCO): This is the most important long-term complication of ECCE with PCIOL. The cause can be primary opacification due to posterior capsular plague, or proliferation of lens epithelium on the capsule (Elschnig's pearls) or arise from capsular fibrosis. The patient presents with painless progressive loss of vision. Clinical examination demonstrates reduction in visual acuity and the opacity is visible under slit lamp examination. Capsulotomy is indicated when there is reduction in vision, PCO obscure fundal view preventing diagnosis of posterior segment disease or when PCO is causing monoocular diplopia. The neodymium: ytrrium-aluminium-garnet laser $(\mathrm{Nd}$ : YAG) laser capsulotomy is the treatment of choice. Because of the post-laser elevation of intra ocular pressure, a drop of apraclonidine $1 \%$ is prophylactically applied before commencing the procedure. The procedure is explained to the patient, consent is obtained, the patient is positioned and topical anesthetic drop is applied. A laser contact lens designed for the procedure is placed on the cornea, the laser settings are adjusted, the aiming beam is targeted on the posterior capsule and shots fired at $30^{\circ}$ angle to reduce astigmatism. A series of shorts in cruciate form are applied avoiding damage to the intraocular lens. An opening of $3 \mathrm{~mm}$ is adequate to improve the patient's vision. Complications include elevation of intraocular pressure. Various reasons have been adduced as to the cause such as blockage of the anterior chamber angle by the fragmented debris and possible inflammation following the procedure. However, the 
pressure elevation tends to be transient and can be control with anti-glaucoma medications. Retinal detachment is another complication which occurs when Nd: YAG capsulotomy is performed early. Early treatment may not be necessary in most instances as PCO tend to occur from two years after surgery, though this is highly influenced by choice of IOL. Ophthalmologists in sub-Saharan Africa generally avoid doing a Nd: YAG capsulotomy earlier than six months after the cataract surgery.

\section{Factors favoring good outcome of surgery}

The proficiency, experience and commitment of the surgeon can partly determine the outcome of surgery. A good surgeon will assess the individual patient, prepare the patient effectively, organize good support services and ensure adequate postoperative care. Thus, selection of suitable patient with appropriate preoperative management is crucial in obtaining good visual outcome. Assessment of the patient involves clinical evaluation for any ocular co-morbidity, or systemic disease that can affect the outcome of surgery. Systemic diseases such as diabetes mellitus and hypertension are known to have retinal and optic nerve complications. Eye diseases such as glaucoma unless detected and without patients being appropriately informed during consent about a possible guarded visual prognosis can be a cause of poor outcome of surgery, dissatisfaction to the patient and a cause of great concern to the ophthalmologist. Silent dacryocystitis can predispose to postoperative endophthalmitis and must where possible be treated before cataract surgery. Patient education is important in reducing harmful eye practices such as application of traditional eye medications after surgery. Postoperative refraction to detect and treat any residual refractive errors is essential in achieving complete rehabilitation of the patient after surgery.

There is need for monitoring of outcome in order to determine causes of poor postoperative vision with the aim of finding ways to prevent them. Better resource allocation and utilization is essential in planning eye care, implementation and evaluation of services to ensure underserved areas are treated -an important part in the path of eradicating avoidable blindness in Africa south of the Sahara.

\section{References}

[1] Abdu L, Foster A. Assessment of resource utilization for the delivery of cataract surgical services Nig J Basic Clin Sci 2005; 2 (1): 1-2

[2] Abdu L. Results of cataract outreach services in a state of Nigeria TAF Prev Med Bull 2010; 9: 225-228

[3] Akinsete E.O; Efficient surgical services for cataracts in Nigeria. Nig J Ophthalmol 1993. $2 ; 4-5$.

[4] American Academy of Ophthalmology. Basic and Clinical Science Course. Section 2: Fundamentals and Principles of Ophthalmology. San Francisco California. 19931994 pp 167-174

[5] Ayanniyi AA, Taoheed AA. Fulfilling human resource development goal in West Africa: can the training of ophthalmologist diplomats be improved? West Afr J Med 2009 May; 28 (3): 177-81 
[6] Briesen S, Geneau R, Roberts H, Opiyo J, Courtright P. understanding why patients with cataract refuse free surgery: the influence of rumors in Kenya. Trop Med Int Health 2010: 15 (5): 534-9

[7] Courtright P, Murenzi J, Mathenge W, Munana J, Muller A. reaching rural Africans with eye services: findings from primary eye care approaches in Rubavu District, Rwanda. Trop Med Int Health 2010 Jun; 15 (6): 692-6

[8] Dean WH, Patel D, Sherwin JC, Metcalfe NH> follow up survey of cataract surgical coverage and barriers to cataract surgery at Nkhoma, Malawi. Ophthalmic Epidemiol 2011 Aug; 18 (4): 171-8

[9] Egbert PR, Buchanan M. Results of extra capsular cataract surgery and intraocular lens implantation in Ghana. Arch Ophthalmol 1991 Dec; 109 (12): 1764-8

[10] Fine BS, Yanoff M: Ocular Histology, ed 2. Hagerstown, MD. Harper \& Row publishers Inc 1979, pp 149-159

[11] Foster A. who will operate Africa's 3 million curably blind people? Lancet 1991 May; 337 (8752): 1267-9

[12] Frick KD, Foster A, Bah M, Faal H. Analysis of cost and benefits of the Gambian Eye Care Program. Arch OIphthalmol 2005 Feb; 123 (2): 239-43

[13] Frimpong-Boateng A, Rufer F, Fiadoyor S, Nkrumah-Mills J, Mensah-Tetteh F, Kudoadzi N, Roider J. [ophthalmological screening via a hospital boat: field study for planning future health care services in remote village at the Volta Lake in Ghana]. Ophthalmologie.2009 Sep; 106 (9): 819-25

[14] Gilbert C, Raman U, Francis V. World Blindness and its prevention. International Agency for Prevention of Blindness. 2005; 7: 67-72

[15] Kyari F, Murthy VSG, Sivsubramaniam S, Gilbert CE, Abdull MM, et al. Prevalence of blindness and visual impairment in Nigeria: The National Blindness and Visual Impairment survey. Invest Ophthalmol Vis Sci 2009; 50 (5): 2033-2039

[16] Limburg H, Foster A, Gilbert C, Johnson GJ, Kyndt M. routine monitoring of visual outcome of cataract surgery. Part 1: Development of an instrument. Br J Ophthalmol 2005 Jan; 89 (1): 45-9

[17] Manual of International Statistical classification if diseases injuries and causes of death. Geneva. WHO. 1997; 53-58.

[18] Ntim-Amponsah CT. Traditional methods of treatment of cataract seen at Korle-Bu Teaching Hospital. West Afr Med J 1995 Apr-Jun; 14 (2): 82-7

[19] Odusote K. human resource development for the prevention of blindness in Anglophone West Africa. West Afr J Med 1998 Jan-Mar; 17 (1): 1-8

[20] Onakpoya OH, Adegbehingbe BO, Adeoye AO, Adewara BA. Ophthalmic admissions in a tertiary hospital in Nigeria. Afri Health Sci 2010 Mar; 10 (1): 54-7

[21] Rabiu MM, Mohammed N. rapid assessment of cataract surgical services in BirninKebbi local government area of Kebbi State, Nigeria. Ophthalmic Epidemiol 2008 Nov-Dec; 15 (6): 359-65

[22] Schemann JF, Bakayoko S, Coulibaly S. traditional couching is not an effective alternative procedure for cataract surgery in Mali. Ophthalmic Epidemiol 2000 Dec; 7(4): $271-83$ 
[23] Thylefors B. The WHO program for the prevention of blindness and cataract in developing countries. Doc Ophthalmol 1992; 81 (3): 339-44 